\title{
Transcription factor CDX2 up-regulates proto-oncogenic miR-744 via a promoter activation mechanism in non-small-cell lung cancer
}

\author{
Zhou Sha ${ }^{1 \#}$, Song $\mathrm{Hao}^{2 \#}$, Fengjiao Long ${ }^{1}$, Yajun $\mathrm{Wei}^{3}$, Shaoyi Chen ${ }^{4}$, Ting $\mathrm{Li}^{1}$, Lina $\mathrm{Yi}^{5}$, Liyang $\mathrm{Hu}^{1}$, \\ Zhong Lin ${ }^{1}$, Jianzhong Xian ${ }^{6}$, Xiaofeng Pei ${ }^{1}$
}

${ }^{1}$ Department of Thoracic Oncology, The Cancer Center of The Fifth Affiliated Hospital of Sun Yat-sen University, Zhuhai, China; ${ }^{2}$ Spinal Surgery Department, Zhuhai People's Hospital (Zhuhai Hospital Affiliated with Jinan University), Zhuhai, China; ${ }^{3}$ Department of Minimally Invasive Interventional Oncology, The Cancer Center of The Fifth Affiliated Hospital of Sun Yat-sen University, Zhuhai, China; ${ }^{4}$ Department of Head and Neck Oncology, Thoracic Oncology, The Cancer Center of The Fifth Affiliated Hospital of Sun Yat-sen University, Zhuhai, China; ${ }^{5}$ Department of Infectious Diseases, The Fifth Affiliated Hospital of Sun Yat-sen University, Zhuhai, China; ${ }^{6}$ Department of Ultrasound, The Fifth Affiliated Hospital of Sun Yat-sen University, Zhuhai, China

Contributions: (I) Conception and design: Z Sha, J Xian, X Pei; (II) Administrative support: None; (III) Provision of study materials or patients: None; (IV) Collection and assembly of data: Z Sha, S Hao, Y Wei; (V) Data analysis and interpretation: Z Sha, S Chen, L Hu, T Li, Z Lin, J Xian, X Pei; (VI) Manuscript writing: All authors; (VII) Final approval of manuscript: All authors.

\#These authors contributed equally to this work.

Correspondence to: Xiaofeng Pei. Department of Thoracic Oncology, The Cancer Center of The Fifth Affiliated Hospital of Sun Yat-sen University, 52 Meihua Road, Zhuhai 519000, China. Email: peixf3@mail.sysu.edu.cn; Jianzhong Xian. Department of Ultrasound, the Fifth Affiliated Hospital of Sun Yat-sen University, 52 Meihua Road, Zhuhai 519000, China. Email: xianjzh@mail.sysu.edu.cn.

Background: The role of caudal-related homeobox 2 (CDX2) in the pathogenesis of non-small cell lung cancer (NSCLC) is unclear. The purpose of this study was to investigate the mRNA (message RNA) expression of CDX2 in NSCLC, and to determine its relationship with miR-744 (microRNA744) and its potential as a biomarker of NSCLC.

Methods: MiR-744 is overexpressed in A549, H460, and H1299 cell lines. Real-time quantitative polymerase chain reaction (qRT-PCR) was used to detect the mRNA expression. A chromatin immunoprecipitation (ChIP) essay was performed to determine the CDX2 binding sites. We then conducted a luciferase reporter essay to analyze interaction between MiR-744 and 3'UTRs (the 3' untranslated sequences). The migration and Boyden chamber method were used to study cell mobility.

Results: In this study, we found that ectopic CDX2 increased the expression of miR-744, while the attenuation of CDX2 reduced the expression of miR-744 by qRT-PCR. Chromatin immunoprecipitation experiments confirmed that CDX2 directly binds to the promoter of miR-744. The luciferase reporter assay further verified the binding sites of -347 to $-358 \mathrm{bp}$ in the most likely promoter like sequence of miR-744. CDX2-induced up-regulation of miR-744 can significantly promote the migration and invasion of NSCLC cells, while overexpression CDX2 is sufficient to rescue the migration and invasion capacity of these cells following knockdown of miR-744.

Conclusions: In summary, our results confirmed for the first time the regulatory mechanism of CDX2 on miR-744 transcription and provided a potential mechanism for CDX2 as an oncogene in lung cancer.

Keywords: Caudal-related homeobox 2 (CDX2); miR-744; non-small cell lung cancer (NSCLC)

Submitted Aug 09, 2021. Accepted for publication Oct 13, 2021.

doi: $10.21037 / \mathrm{atm}-21-4526$

View this article at: https://dx.doi.org/10.21037/atm-21-4526 


\section{Introduction}

Lung cancer has gradually become the leading malignancy worldwide in terms of its rates of morbidity $(11.6 \%)$ and mortality (18.4\%) (1). Metastasis occurs in more than $70 \%$ of patients and accounts for more than $90 \%$ of cancerrelated deaths (2). Increasing evidence supports the participation of microRNAs (miRNA) in the metastatic process of cancers, including lung cancer, by negatively or positively regulating the expression of metastasis associated genes through post-transcriptional repression, mRNA (message RNA) degradation, or promoter activation $(3,4)$.

Recently, miR-744 (microRNA744) was reported as a tumor-related gene, and its dysregulation was identified in serum or specimens of patients with head and neck cancers, multiple myeloma, gastric cancer, hepatocellular carcinoma, or breast cancer (3-7). In our previous study, we reported that miR-744 expression was elevated in nasopharyngeal carcinoma (NPC) specimens and was significantly associated with NPC progression, and confirmed for the first time that miR-744 participates in the process of cancer metastasis as a proto-oncogene (8). Furthermore, we identified miR-744 as an up-regulated miRNA in a non-small-cell lung cancer (NSCLC) cell line, and found that miR-744 up-regulation could significantly promote the migration and invasion of both NPC and NSCLC cells (9).

There are several regulatory mechanisms of miRNA expression, such as defects in the biogenesis machinery of miRNAs, structural genetic alterations, epigenetic changes, altered transcription factor (TF) activity, and so on (10). In our previous study, we identified that TF c-Jun directly binds to the miR-744 promoter region, resulting in elevated miR-744 expression in both NPC and NSCLC cells (9).

Also, bioinformatics analysis (PROMO 2.0) revealed that the 2-kb region upstream of the miR-744 gene contains three potential binding motifs for TF caudaltype homeobox transcription factor 2 (CDX2). CDX2 is expressed in the nuclei of intestinal epithelial cells and is essential for embryonic formation and differentiation of the intestine; reduced expression of $\mathrm{CDX} 2$ can result in metastatic tumors (11). The loss of CDX2 synergizing with the loss of NKX2-1 was reported to fully activate the metastatic program of lung adenocarcinoma, and were consistently down-regulated in metastatic cells compared with non-metastatic cells (12). Previous study has reported the mRNA expression of CDX2 is detectable at a high frequency in the tumor and normal tissue of patients with non-small cell lung cancer (13). However, the function and mechanism of CDX2 in NSCLC is still unknown. CDX2 and hindfoot or pituitary homologous frame gene 1 (BFT or PITX1) are members of the homologous frame family. In addition, BFT expression plays a critical role in lung development (14). A recent study reported that BFT expression may be associated with the development of lung cancer (15).

The aim of this study was to investigate the mRNA expression of CDX2 in NSCLC, and to determine its relationship with miR-744 and its potential as a biomarker of NSCLC. We present the following article in accordance with the MDAR reporting checklist (available at https:// dx.doi.org/10.21037/atm-21-4526).

\section{Methods}

\section{Cell lines and cell culture}

Human SPC-A-1 and A549 cells were purchased from the Shanghai Cell Bank of Chinese Academy of Science (Shanghai, China) and maintained in RPMI-1640 medium (Roswell Park Memorial Institute 1640 medium) (Corning, NY, USA) with $10 \%$ fetal bovine serum (FBS, GIBCO, South America, NY, USA). All cells were incubated in a $5 \%$ carbon dioxide $\left(\mathrm{CO}_{2}\right)$ atmosphere at $37^{\circ} \mathrm{C}$.

\section{Plasmid and oligonucleotide construction}

The miR-744 mimics, inhibitor, and its negative control miRNA (anti-miR-control) were designed and synthesized by Genepharma (Shanghai, China) based on the miRbase database (http://www.miRbase.org). The sequences of miR-744-mimic and inhibitor are as followed: 5 '-CUGUUAGCCCUAGCCCCGCAUU-3', for miR744-mimic; 5'-UGCGGGGCUAGGGCUAACAGCA-3', for miR-744-inhibitor. Additionally, the siRNA (Small interfering RNA) against CDX2 and non-specific siRNA control were also designed and synthesized by Genepharma (Shanghai, China). The plasmids expressing CDX2 and negative control pENTER were designed and synthesized by Vigene Bioscience (Shandong, China).

\section{Transient transfection}

The SPC-A-1 and A549 cells were seeded $\left(2 \times 10^{5}\right.$ per well) in six-well plates at $30-40 \%$ density. Transient transfection was performed using Lipofectamine 3000 reagent (Invitrogen, USA). The cells were then incubated at 5\% 
$\mathrm{CO}_{2}$ and $37^{\circ} \mathrm{C}$ and collected $48 \mathrm{~h}$ after transfection.

\section{$R N A$ extraction and quantitative real-time PCR (qRT-PCR) analyses}

Total RNA was extracted from the cell lines using TRIzol $^{\circledR}$ Reagent (Invitrogen, CA, USA) according to the manufacturer's recommended protocol. For detection of miR-744 mRNA, total RNA was reverse transcribed into cDNA (complementary DNA) to perform qRT-PCR using the SYBR ${ }^{\circledR}$ PrimeScript $^{\mathrm{TM}}$ miRNA RT-PCR Kit (TaKaRa, Dalian, China). For detection of CDX2 mRNA, total RNA was reverse transcribed into cDNA using the PrimeScript ${ }^{\mathrm{TM}}$ RT reagent Kit (TaKaRa, Dalian, China). qRT-PCR for CDX2 mRNA was performed in a Mx3005P real-time PCR instrument (Stratagene, USA) using SYBR® Premix Ex Taq $^{\text {TM }}$ (TaKaRa, Dalian, China). Small nuclear RNA U6 (U6 snRNA) or glyceraldehyde-3-phosphate dehydrogenase (GAPDH) was used as internal control for miRNA or mRNA expression, respectively.

The sequences of the primers are shown as follows: hsamiR-744 forward: 5'-ACACTCCAGCTGGGTGCGGG GCTAGGGCTAAC-3', U6 forward: 5'-GCTTCGGCA GCACATATACTAAAAT-3'. MiR universal reverse prime: 5'-CTCAACTGGTGTCGTGGA-3', CDX2 forward: 5 ' UCCUCUGAGAAGUGUCCCATT-3', reverse: 5'UGGGACACUUCUCAGAGGATT-3', GAPDH forward: 5'-GCACCGTCAAGGCTGAGAAC-3', reverse: 5'-TGGTGAAGACGCCAGTGGA-3'. The relative expression of miR-744 and CDX2 was calculated according to the $2^{-\Delta \Delta \mathrm{Ct}}$ method.

\section{Promoter reporter construction and luciferase assays}

The upstream of mir-744 gene was amplified by qPCR in different length regions. A mutation primer was designed for CDX2 conjugate sequences mismatched $12 \mathrm{bp}$ in -347 to -358 . The sequences built by these promoters are listed in Table S1. The amplified products were cloned into the pGL3 base vector to produce the promoter builder of mir744. The SPC-A-1 and A549 cells were transfected into a 24-well plate at a density of $5 \times 10^{3}$ cells $/ 100 \mathrm{ng}$, A mixture of $150 \mathrm{ng}$ promoter constructs (Luc-miR-744-A, Luc-miR744-B and Luc-miR-744-C) or control vector, $100 \mathrm{nmol}$ mimic-miR-744 or mimic-NC, and $5 \mathrm{ng}$ pRL-TK vector (Promega, WI, USA) were co-transfected into SPC-A-1 and A549 cells cells per well using Lipofectamine 2000 and incubated for 48 hours. A double luciferase reporter gene detection system (Promega, WI, USA) was used to detect luciferase according to the manufacturer's instructions.

\section{Cell migration and invasion assays}

Cell invasion experiments were carried out in a 24-well plate using a transwell chamber (Corning, NY, USA). At $48 \mathrm{~h}$ after transfection, each group of cells $\left(10^{5}\right.$ cells/100 largose) were resuspended in serum-free medium, inoculated in the upper chamber with $5 \%$ matrix coated membrane (BD Bioscience, San Jose, CA, USA), and the lower chamber was filled with fresh and complete medium of 500 largose. After incubation at $37{ }^{\circ} \mathrm{C}$ with $5 \% \mathrm{CO}_{2}$ for $24 \mathrm{~h}$, the noninvasive cells were scraped from the upper surface of the filter with a cotton swab. The invading cells adhering to the lower surface of the lumen were fixed with methanol and stained with hematoxylin. An inverted microscope (EVOSM500, Central Laboratory of Southern Medical University) was used to manually count the invading cells at 200x magnification in three random fields. Similar nonmatrix inserts were used for the migration experiments.

\section{Wound bealing assay}

At the stage when the cells had grown to approximately $90 \%$ fusion (48 h after transfection), an artificial wound was created with a $20-\mu \mathrm{L}$ pipette head. The cells were then cultured in a fresh medium. Images were taken at 0 and $36 \mathrm{~h}$, respectively to observe wound healing. The relative percentage of the wound healing was calculated as follows: ( $0 \mathrm{~h}$ wound width $-36 \mathrm{~h}$ wound width $) / 0 \mathrm{~h}$ wound width.

\section{Statistical analysis}

Data were expressed as the mean \pm standard error of triplicates. Each experiment was repeated at least three times. Statistical analyses were performed using SPSS 13.0 (SPSS Inc., Chicago, USA). The Student's $t$-test was used to assess differences between groups in in vitro studies. In all cases, $\mathrm{P}<0.05$ was considered to be statistically significant.

\section{Results}

\section{CDX2 is overexpressed in NSCLC cell lines}

As shown in Figure 1, the expression of CDX2 was significantly increased in human NSCLC cell lines compared with the normal lung epithelial cell line (HBE). 


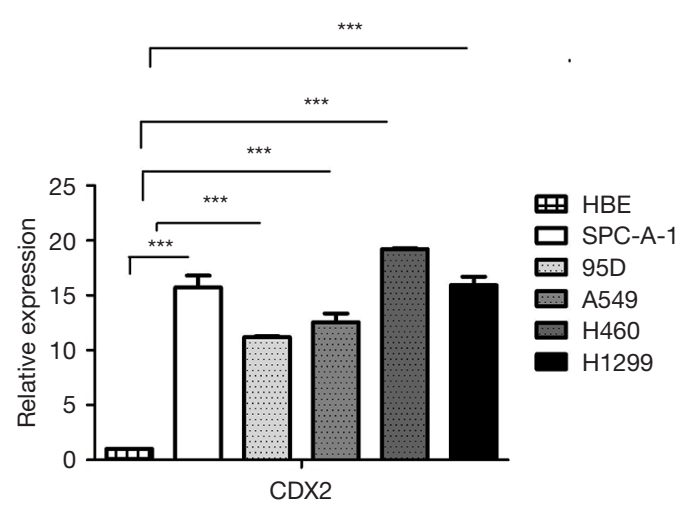

Figure 1 CDX2 expression is up-regulated in human NSCLC cell lines. QRT-PCR analysis was performed to quantify CDX2 expression in A549, H460, H1299, SPC-A-1, 95D, and normal lung epithelial (HBE) cell lines. ${ }^{* * *}, \mathrm{P}<0.001$ compared to controls.

\begin{tabular}{|c|c|c|c|c|}
\hline CDX & 2 [Т03246] & was predi & d in: & \\
\hline Sequence & CAAGCACAT & AAA TTAAT & ATAAA TTTA & ATCCC \\
\hline & 347 & 3581186 & 11971232 & 1243 \\
\hline Dissimilarity & $14.34 \%$ & & & \\
\hline RE equally & 0.05150 & & & 907 \\
\hline RE query & 0.15926 & & & 458 \\
\hline
\end{tabular}

Consensus sequence and matrix:

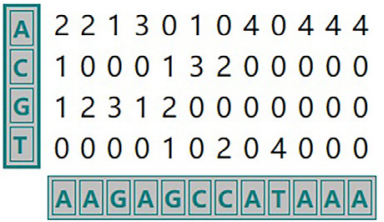

Figure 2 Bioinformatics analysis by PROMO 2.0 for possible transcription factor binding sites (TFBS) in the miR-744 3'UTR promoter region.

Also, the level of CDX2 was markedly higher in A549, H460, and H1299 cells compared to SPC-A-1 and 95D cells. Furthermore, the cell migration and invasion capacity was also higher in NSCLC cell lines with high CDX2 levels, which suggested that CDX2 may play a role in the migration and invasion of NSCLC.

\section{CDX2 up-regulated miR-744 expression in NSCLC cell lines}

To identify the potential TFs regulating miR-744
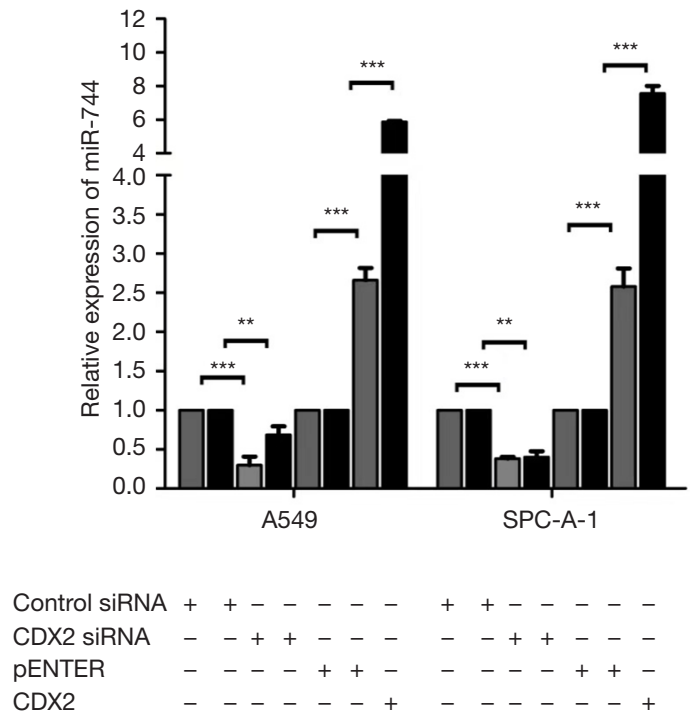

Figure 3 CDX2 activated miR-744 transcription. The expression of CDX2-mediated miR-744 was quantified by qRT-PCR analysis in A549 and SPC-A-1 cells. GAPDH or U6 was used as internal control. **, $\mathrm{P}<0.01$; *** $\mathrm{P}<0.001$ compared to controls.

transcription, we used PROMO 2.0 to search for possible transcription factor binding sites (TFBS) in the miR-744 3'UTR promoter region (Figure 2). The bioinformatics analysis showed that the $2-\mathrm{kb}$ region upstream of the miR744 gene contains three potential binding sides for the TF CDX2.

To confirm the bioinformatics prediction and explore the relationship between CDX2 and miR-744, we used CDX2 siRNA, CDX2 plasmid, and their controls to transiently transfect into A549 and SPC-A-1 cells. The expression of CDX2 mRNA was measured by qRT-PCR to detect the efficiency of transfection (Figure 3). The results showed that the transfection of CDX2 siRNA led to significant knockdown of miR-744 expression (Figure 3), while the overexpression of CDX2 led to a marked up-regulation of miR-744 in NSCLC cell lines (Figure 3).

To further determine whether CDX2 could affect the endogenous expression of miR-744 in these cells, we performed a co-transfection of CDX2 plasmid, miR-744 mimics, inhibitor, and controls in A549 and SPC-A-1 cells. The existence of CDX2 can reverse the knockdown of miR744 and intensify the effect of miR-744 mimics (Figure 3). Taken together, these results suggested that CDX2 plays an extremely important role in miR-744 transcription regulation. 


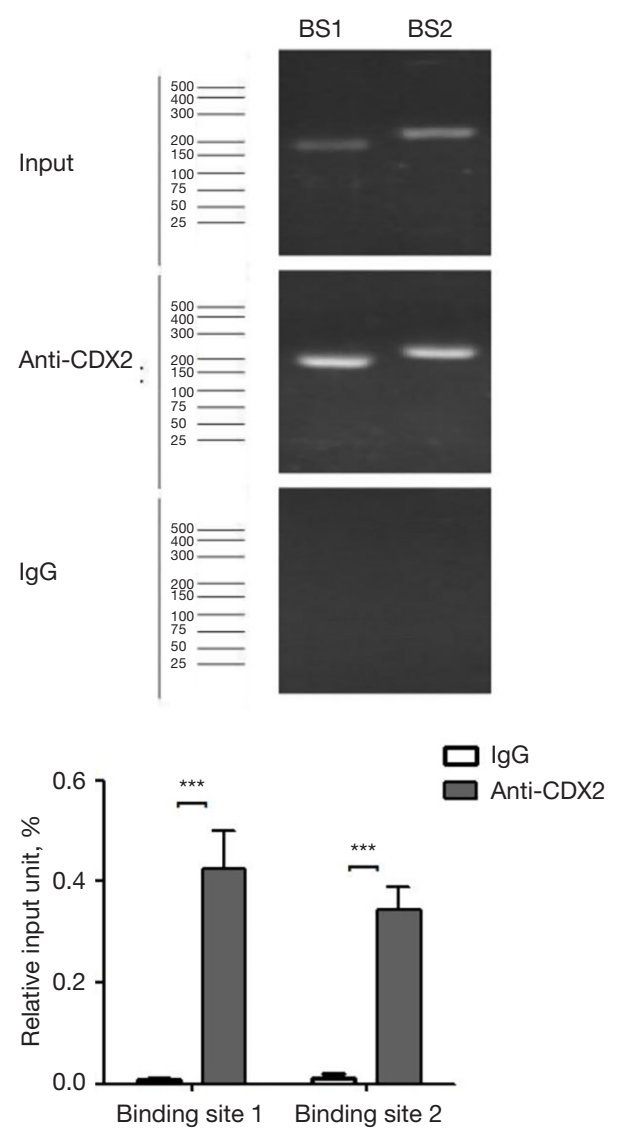

Figure $4 \mathrm{CDX} 2$ can be recruited to bind to the promoter region of miR-744. (A) In A549 cells transfected with a vector expressing CDX2, chromatin immunoprecipitation experiments were performed to identify the CDX2 binding sites on the miR-744 promoter. An isotype-matched IgG matched with the same type was used as negative control. (B) Two binding sites in the miR-744 promoter were analyzed by qRT-PCR using specific primers. ${ }^{* * *}$, $\mathrm{P}<0.001$ compared to controls. BS, binding site.

\section{CDX2 is recruited to binding to the promoter region of miR-744}

TFs can bind to either the enhancer or promoter regions of DNA adjacent to the genes that they regulate (15). We performed a chromatin immunoprecipitation (ChIP) assay coupled with qRT-PCR to determine whether CDX2 directly binds to its potential promoter region, as predicted in the bioinformatics analysis, to up-regulate the transcription of miR-744.

We designed primers for the ChIP assay based on the predicted CDX2 binding sites in the miR-744 potential promoter region, which were -347 to $-358 \mathrm{bp},-1,186$ to
$-1,197 \mathrm{bp},-1,232$ to $-1,243 \mathrm{bp}$, and took the percentage of similarity between the CDX2 DNA-binding domains (DBDs) into consideration.

Since the minimum amplicon size for TFBS is approximately 150 base pairs in qRT-PCR, it is technically difficult to design primers to amplify $-1,186$ to $-1,197 \mathrm{bp}$ and $-1,232$ to $-1,243$ bp regions separately. Therefore, we amplified these two binding sites as one binding region, which was renamed to Site 1 in our previous study (9). The remaining -347 to -358 bp were named Site 2 .

As shown in Figure 4A, CDX2 could bind to Site 1 and Site 2 sequences in the miR-744 potential promoter region in A549 cells, which was validated by quantitative ChIP assays (Figure 4B). An isotype-matched immunoglobulin $\mathrm{G}(\mathrm{IgG})$ was used as a negative control. These results demonstrated that CDX2 could be recruited to the three binding sites ( -347 to $-358 \mathrm{bp},-1,186$ to $-1,197 \mathrm{bp}$, and $-1,232$ to $-1,243 \mathrm{bp}$ ) in the miR-744 potential promoter region.

\section{CDX2 response element existed in the key promoter region of $m i R-744$}

In our previous study, we found that the most potential promoter-like sequence of miR-744 existed in the -500 to -1 bp region (9). In order to verify the presence of CDX2 response elements in the key promoter region of miR744 , we conducted co-transfection studies. Luciferase activity was detected $48 \mathrm{~h}$ after transfection with the miR744 promoter (-500 to $-1 \mathrm{bp}$ ), empty pGL3-basic vector and CDX2 plasmid, or corresponding empty vector in SPC-A-1 and A549 cells (Figure 5). Accordingly, the miR744 promoter construction with only $-500 \mathrm{bp}$ resulted in a significant increase in relative luciferase activity in SPC-A-1 and A549 cells, which was further strengthened 2-18 times, suggesting the functional promoter activation region may exists in the upstream of $-500 \mathrm{bp}$ CDX2 miR-744 gene.

\section{MiR-744 mediates CDX2 to promote lung cancer cell migration and invasion}

We previously reported that the overexpression of miR744 can up-regulate the migration and invasion of NSCLC cells (9). To investigate whether the cell functions induced by CDX2 could be mediated by miR-744, CDX2 plasmids and the miR-744 inhibitor were co-transfected into SPC-A-1 and A549 cells. Q-PCR results showed forced expression of CDX2, and overexpression of CDX2 led to markedly 


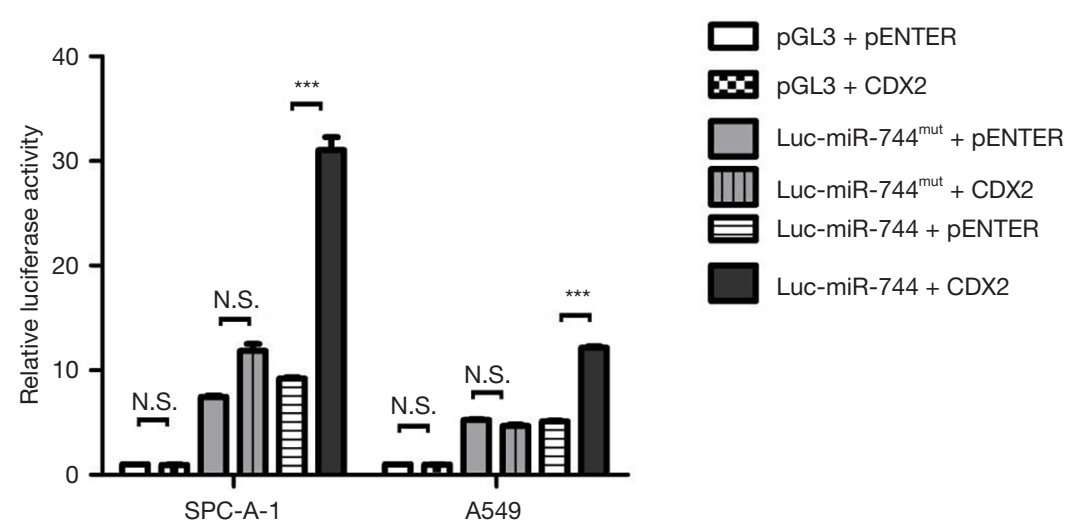

Figure 5 CDX2 response element existed in the key promoter region of miR-744. The luciferase activity of the miR-744 promoter reporter construct and empty pGL3-based vector were measured in SPC-A-1 and A549 cells in the presence or control of CDX2 plasmid. ***, $\mathrm{P}<0.001$ compared to controls. NS, non-significant.

increased expression of mir-744 compared with the control group (Figure 6A). Accordingly, the mir-744 inhibitor reduced miR-744 expression, and was rescued by CDX2 to normal levels (Figure 6A).

Subsequently, we studied whether the miR-744 inhibitor could offset the CDX2 promotion of cancer cell migration and invasion. Using scratch and transwell migration and invasion experiments, it was observed that CDX2 overexpression could significantly promote the migration and invasion of SPC-A-1 and A549 cells (Figure 6B-6E), while the positive effects of CDX2 on migration and invasion were almost completely suppressed by miR-744 inhibitor. These results showed that CDX2 increased the expression of miR-744 and participated in the promotion of migration and invasion.

\section{Discussion}

MiRNAs are highly conserved, non-coding, small RNA molecules that can play a dual role in tumor cell apoptosis, proliferation, angiogenesis, invasion, and metastasis (14). MiR-744 has been shown to participate in tumor invasion and migration as a tumor promoter or suppressor. In our previous miRNA microarray analysis, we found that miR744 is markedly decreased after MTA1 gene knockdown (15), and we confirmed that miR744 functions as proto-oncogene in human NPC progression and metastasis (9). Recently, we reported the oncogenic functions of miR-744 in the promote invasion and migration in NPC and NSCLC. Furthermore, in that study, we found that c-Jun-induced up-regulation of miR-744 mediated the effects of c-Jun signaling in NPC and
NSCLC, providing a new insight into oncogenic functions of miR-744.

Caudal-type homeobox 2 (CDX2) is an intestine-specific TF with potential prognostic and predictive effects. Also, hindfoot or pituitary homologous frame gene 1 (BFT or PITX1) are members of the homologous frame family, and nuclear TFs play an important role in regulating intestinal epithelial cell proliferation and differentiation in fetal and adult tissues (16-18). CDX2 has been proposed as a potential molecular marker in several malignancies, including gastric, colon, and esophageal cancers (19-21). In addition, CDX2 expression has been studied as a reliable marker of colorectal origin for the determination of pulmonary metastasis $(22,23)$. Recent reports have indicated that CDX2 expression is not limited to metastases of colorectal origin, but can also be detected in primary lung adenocarcinoma by RT-PCR and immunohistochemistry $(24,25)$. In this study, CDX2 expression was consistent in primary lung adenocarcinoma.

The relationship between CDX2 expression and cancer occurrence has been less studied. For the first time, our team reported that miR-744, as an up-regulated miRNA in NSCLC cell lines, can be up-regulated via a promoter activation mechanism (9). Recently, Chen et al. confirmed for the first time changes in BFT expression in primary lung cancer, suggesting that BFT may be associated with the occurrence and development of lung cancer (15).

Also, for the first time in this study, we demonstrated that the TF CDX2 plays an important role in the transcription of miR-744. CDX2 was the target gene of miR-744 and miR-744 can be directly induced by the tumor promoter 
A

SPC-A-1

$\square$ A549

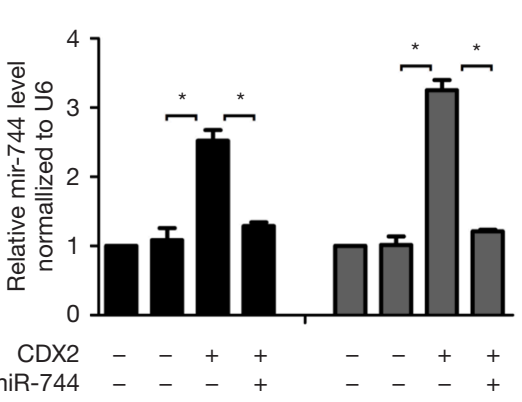
anti-miR-744
C SPC-A-1

며 BLANK
므
CDX2
CDX2 + anti-miR-744

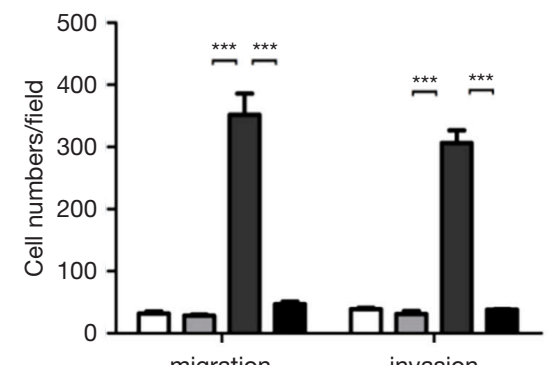

migration invasion
A549

ㅁ BLANK

口NC

口 CDX2

- CDX2 + anti-miR-744
B

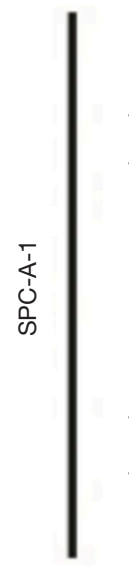

过

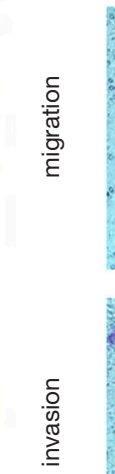
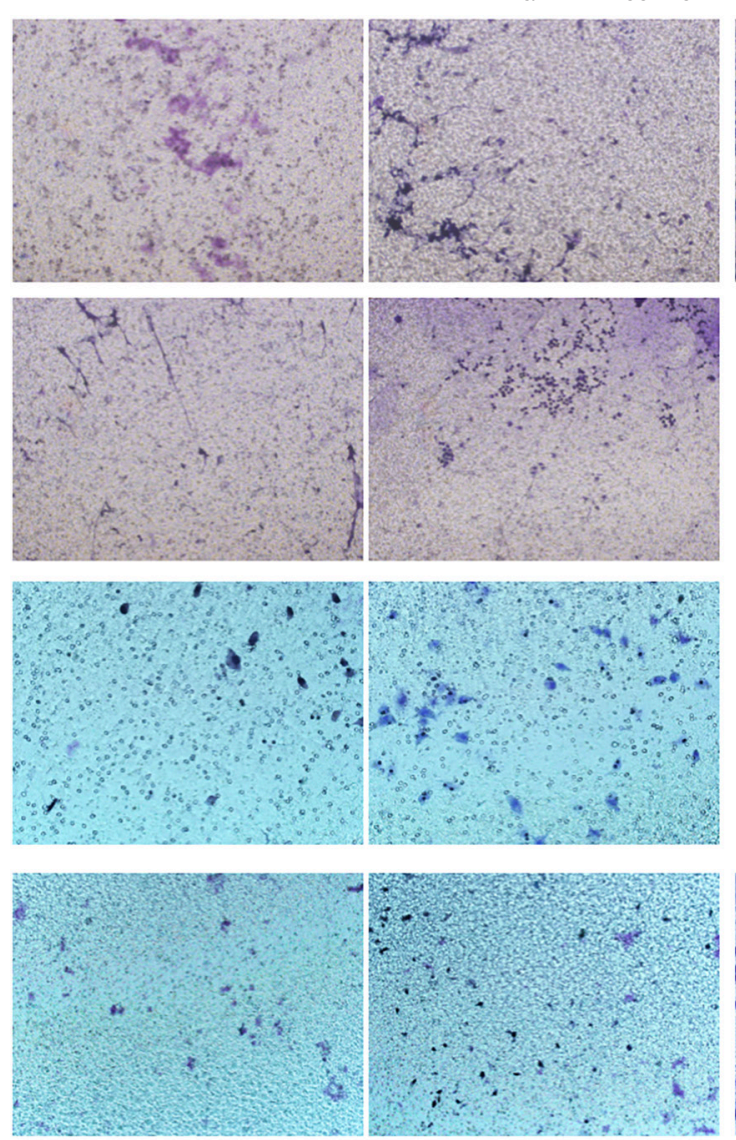

CDX2 + anti-miR-control
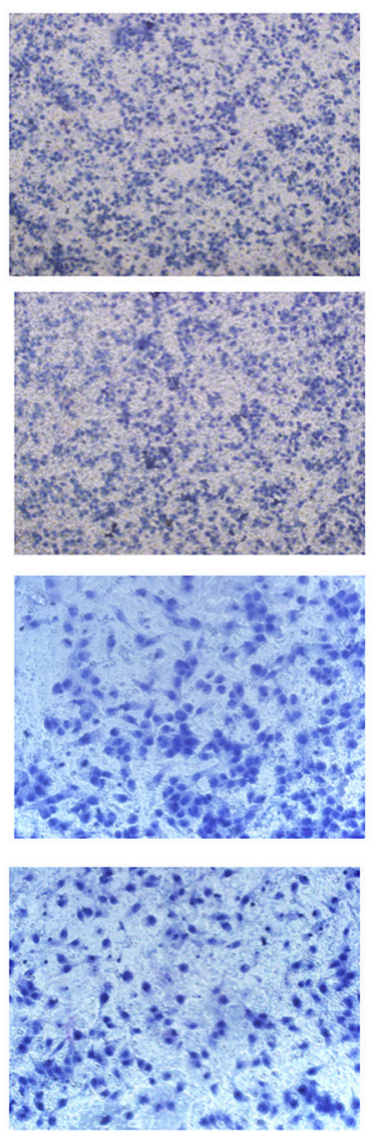

CDX2 + anti-miR-744
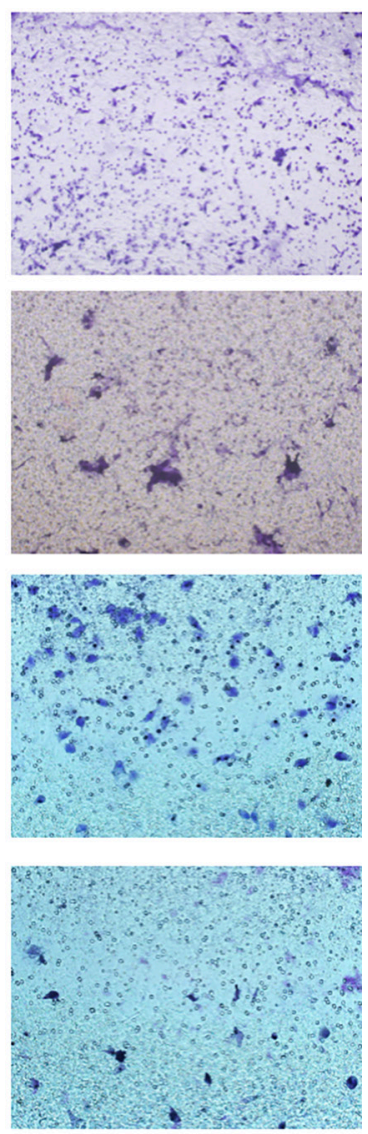
D
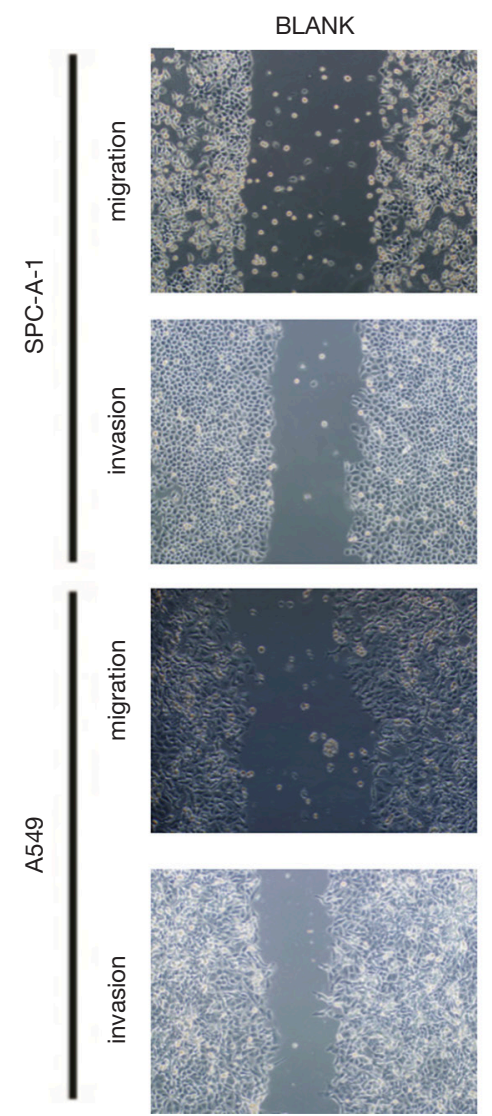

E
PENTER + anti-miR-control
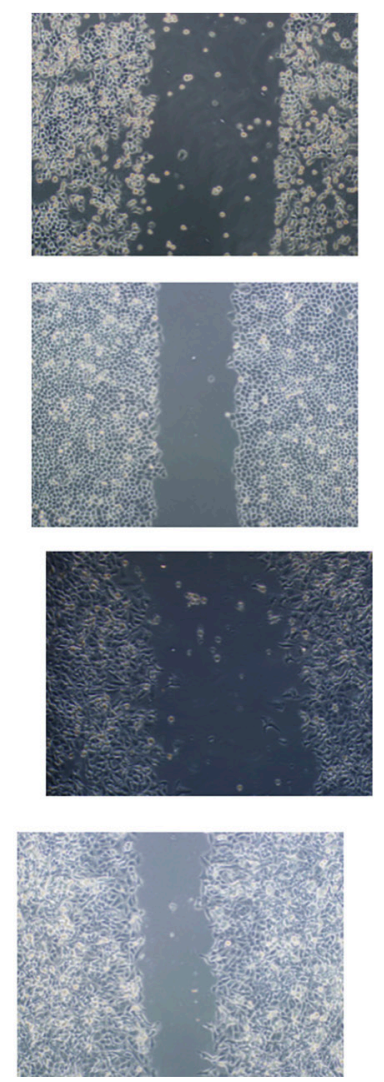

CDX2 + anti-miR-744
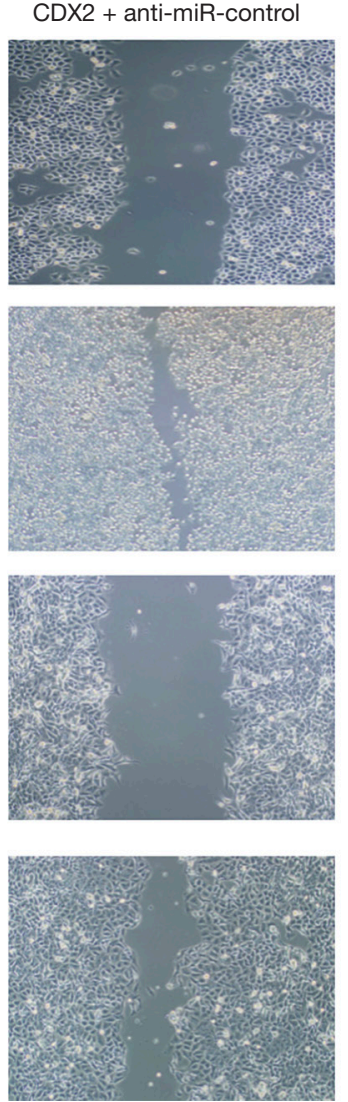

A549

口 BLANK

$\square$ pGL3 + anti-miR-744-control

GDX2 + anti-miR-744-control

- CDX2 + anti-miR-744

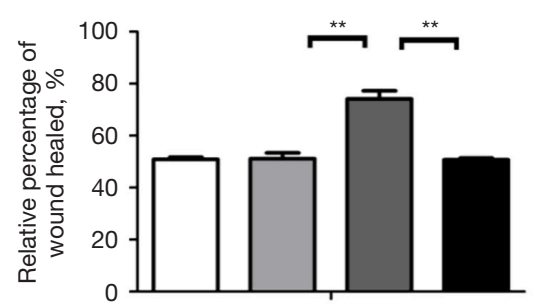

Figure 6 MiR-744 mediates CDX2 to promote lung cancer cell migration and invasion. (A) QRT-PCR was used to analyze the miR744 inhibitor or its vector control and CDX2 plasmid transfection of SPC-A-1 and A549 cells. (B,C) CDX2 promoted the migration and invasion of SPC-A-1 and A549 cells. The miR-744 inhibitor almost completely diminished the positive role of CDX2. Transwell, Boyden, and $(\mathrm{D}, \mathrm{E})$ wound healing methods were used. *, $\mathrm{P}<0.05$ compared to controls; ${ }^{* *}, \mathrm{P}<0.01$ compared to controls; ${ }^{* * *}, \mathrm{P}<0.001$ compared to controls. Original magnification: $\times 200$, the invading cells were stained with hematoxylin.

CDX2 and is partly involved in the invasive phenotype induced by this oncogene. We also identified the upstream -347 to -358 of miR-744 gene as a directly effective CDX2 binding site in the miR-744 promoter region. In addition, our data revealed for the first time the role of CDX2 in promoting migration and invasion in NSCLC.

In summary, the data presented in this paper provide insights into the mechanism of CDX2 dysregulation, and 
establish a direct link between CDX2 signals and miRNAs during dysregulation. Advances in the mechanism of upregulation of CDX2 expression in cancer have laid a theoretical foundation for determining when and how targeted anti-CDX2 and miR-744 therapies are feasible. The current mechanisms provide new insights into the carcinogenic functions of CDX2 and a better understanding of the up-regulation of CDX2 in cancer. CDX2-induced miR-744 up-regulation mediates the role of CDX2 signaling in NSCLC. Further studies on the role and mechanism of CDX2 in NSCLC will be summarized in our next study.

\section{Acknowledgments}

An earlier version of this manuscript has been presented as preprint in research square according to the following link: https://www.researchsquare.com/article/rs-127480/v1.

Funding: This work was funded by the Zhuhai Science and Technology Planning Project (ZH2202200004HJL) from The Fifth Affiliated Hospital of Sun Yat-sen University.

\section{Footnote}

Reporting Checklist: The authors have completed the MDAR reporting checklist. Available at https://dx.doi. org/10.21037/atm-21-4526

Data Sharing Statement: Available at https://dx.doi. org/10.21037/atm-21-4526

Conflicts of Interest: All authors have completed the ICMJE uniform disclosure form (available at https://dx.doi. org/10.21037/atm-21-4526). The authors have no conflicts of interest to declare.

Ethical Statement: The authors are accountable for all aspects of the work in ensuring that questions related to the accuracy or integrity of any part of the work are appropriately investigated and resolved.

Open Access Statement: This is an Open Access article distributed in accordance with the Creative Commons Attribution-NonCommercial-NoDerivs 4.0 International License (CC BY-NC-ND 4.0), which permits the noncommercial replication and distribution of the article with the strict proviso that no changes or edits are made and the original work is properly cited (including links to both the formal publication through the relevant DOI and the license). See: https://creativecommons.org/licenses/by-nc-nd/4.0/.

\section{References}

1. Jemal A, Siegel R, Ward E, et al. Cancer statistics, 2008. CA Cancer J Clin 2008;58:71-96.

2. Ma L, Teruya-Feldstein J, Weinberg RA. Tumour invasion and metastasis initiated by microRNA-10b in breast cancer. Nature 2007;449:682-8.

3. Nurul-Syakima AM, Yoke-Kqueen C, Sabariah AR, et al. Differential microRNA expression and identification of putative miRNA targets and pathways in head and neck cancers. Int J Mol Med 2011;28:327-36.

4. Kubiczkova L, Kryukov F, Slaby O, et al. Circulating serum microRNAs as novel diagnostic and prognostic biomarkers for multiple myeloma and monoclonal gammopathy of undetermined significance. Haematologica 2014;99:511-8.

5. Song MY, Pan KF, Su HJ, et al. Identification of serum microRNAs as novel non-invasive biomarkers for early detection of gastric cancer. PLoS One 2012;7:e33608.

6. Tan YL, Bai ZG, Zou WL, et al. miR-744 is a potential prognostic marker in patients with hepatocellular carcinoma. Clin Res Hepatol Gastroenterol 2015;39:359-65.

7. Leivonen SK, Sahlberg KK, Mäkelä R, et al. Highthroughput screens identify microRNAs essential for HER2 positive breast cancer cell growth. Mol Oncol 2014;8:93-104.

8. Zhu X, Zhang X, Wang H, et al. MTA1 gene silencing inhibits invasion and alters the microRNA expression profile of human lung cancer cells. Oncol Rep 2012;28:218-24.

9. Sha Z, Zhu X, Li N, et al. Proto-oncogenic miR-744 is upregulated by transcription factor c-Jun via a promoter activation mechanism. Oncotarget 2016;7:64977-86.

10. Fang Y, Zhu X, Wang J, et al. MiR-744 functions as a proto-oncogene in nasopharyngeal carcinoma progression and metastasis via transcriptional control of ARHGAP5. Oncotarget 2015;6:13164-75.

11. Asgari-Karchekani S, Karimian M, Mazoochi T, et al. CDX2 Protein Expression in Colorectal Cancer and ItsCorrelation with Clinical and Pathological Characteristics, Prognosis, and Survival Rate of Patients. J Gastrointest Cancer 2020;51:844-9.

12. Li CM, Gocheva V, Oudin MJ, et al. Foxa2 and Cdx2 cooperate with $\mathrm{Nkx} 2-1$ to inhibit lung adenocarcinoma 
metastasis. Genes Dev 2015;29:1850-62.

13. Grimminger P, Ling FC, Neiss S, et al. The role of the homeobox genes BFT and CDX2 in the pathogenesis of non-small cell lung cancer. Anticancer Res 2009;29:1281-6.

14. Gage PJ, Suh H, Camper SA. Dosage requirement of Pitx2 for development of multiple organs. Development 1999; 126:4643-51.

15. Chen Y, Knösel T, Ye F, et al. Decreased PITX1 homeobox gene expression in human lung cancer. Lung Cancer 2007;55:287-94.

16. Walters JR, Howard A, Rumble HE, et al. Differences in expression of homeobox transcription factors in proximal and distal human small intestine. Gastroenterology 1997;113:472-7.

17. Beck F, Chawengsaksophak K, Waring P, et al. Reprogramming of intestinal differentiation and intercalary regeneration in $\mathrm{Cdx} 2$ mutant mice. Proc Natl Acad Sci U S A 1999;96:7318-23.

18. Tamai Y, Nakajima R, Ishikawa T, et al. Colonic hamartoma development by anomalous duplication in Cdx2 knockout mice. Cancer Res 1999;59:2965-70.

19. Barbareschi M, Murer B, Colby TV, et al. CDX2 homeobox gene expression is a reliable marker of colorectal adenocarcinoma metastases to the lungs. Am J Surg Pathol 2003;27:141-9.

Cite this article as: Sha Z, Hao S, Long F, Wei Y, Chen S, Li T, Yi L, Hu L, Lin Z, Xian J, Pei X. Transcription factor CDX2 up-regulates proto-oncogenic miR-744 via a promoter activation mechanism in non-small-cell lung cancer. Ann Transl Med 2021;9(20):1538. doi: 10.21037/atm-21-4526
20. Lord RV, Brabender J, Wickramasinghe K, et al. Increased CDX2 and decreased PITX1 homeobox gene expression in Barrett's esophagus and Barrett's-associated adenocarcinoma. Surgery 2005;138:924-31.

21. Werling RW, Yaziji H, Bacchi CE, et al. CDX2, a highly sensitive and specific marker of adenocarcinomas of intestinal origin: an immunohistochemical survey of 476 primary and metastatic carcinomas. Am J Surg Pathol 2003;27:303-10.

22. Saad RS, Cho P, Silverman JF, et al. Usefulness of Cdx2 in separating mucinous bronchioloalveolar adenocarcinoma of the lung from metastatic mucinous colorectal adenocarcinoma. Am J Clin Pathol 2004;122:421-7.

23. Tanaka S, Saito K, Ito T, et al. CDX2 as a useful marker of colorectal adenocarcinoma metastases to lung in preoperative biopsy specimens. Oncol Rep 2007;18:87-92.

24. Moskaluk CA, Zhang H, Powell SM, et al. Cdx2 protein expression in normal and malignant human tissues: an immunohistochemical survey using tissue microarrays. Mod Pathol 2003;16:913-9.

25. Yatabe Y, Koga T, Mitsudomi T, et al. CK20 expression, CDX2 expression, K-ras mutation, and goblet cell morphology in a subset of lung adenocarcinomas. J Pathol 2004;203:645-52.

(English Language Editor: A. Kassem) 
Table S1 The sequences of the promoter constructs in promoter activity assay

\begin{tabular}{|c|c|}
\hline Promoter constructs & Sequence \\
\hline Luc-miR-744-A & 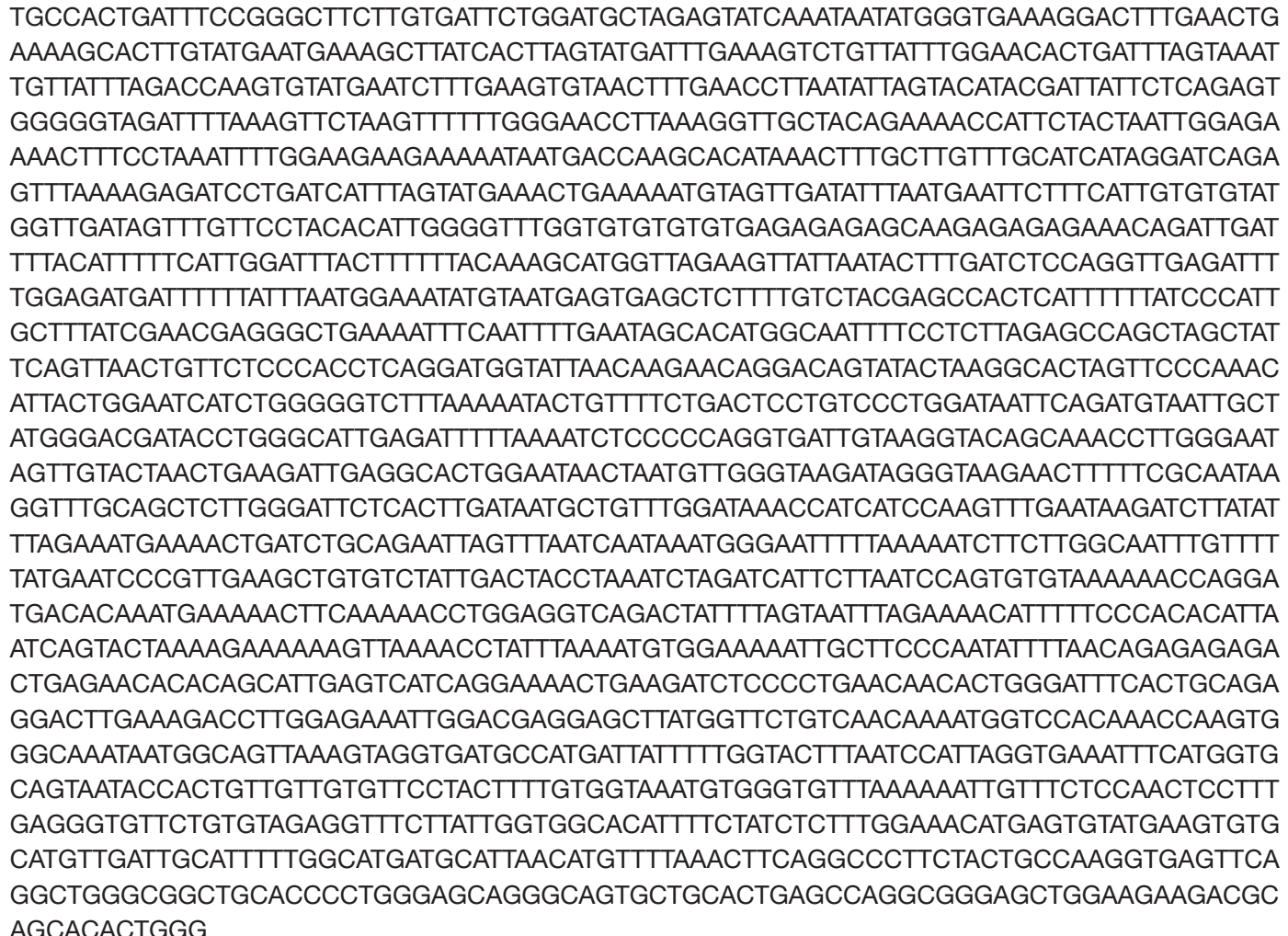 \\
\hline
\end{tabular}

Luc-miR-744-B AGTTGTACTAACTGAAGATTGAGGCACTGGAATAACTAATGTTGGGTAAGATAGGGTAAGAACTTTITCGCAATAA GGTTTGCAGCTCTTGGGATTCTCACTTGATAATGCTGTTTGGATAAACCATCATCCAAGTTTGAATAAGATCTTATA TTTAGAAATGAAAACTGATCTGCAGAATTAGTTTAATCAATAAATGGGAATTTTTAAAAATCTTCTTGGCAATTGTTT TTATGAATCCCGTTGAAGCTGTGTCTATTGACTACCTAAATCTAGATCATTCTTAATCCAGTGTGTAAAAAACCAGG ATGACACAAATGAAAAACTTCAAAAACCTGGAGGTCAGACTATTTTAGTAATTTAGAAAACATTTTTCCCACACATT AATCAGTACTAAAAGAAAAAAGTTAAAACCTATTTAAAATGTGGAAAAATTGCTTCCCAATATTTTAACAGAGAGAG ACTGAGAACACACAGCATTGAGTCATCAGGAAAACTGAAGATCTCCCCTGAACAACACTGGGATTTCACTGCAG AGGACTTGAAAGACCTTGGAGAAATTGGACGAGGAGCTTATGGTTCTGTCAACAAAATGGTCCACAAACCAAGT GGGCAAATAATGGCAGTTAAAGTAGGTGATGCCATGATTATITTGGTACTTTAATCCATTAGGTGAAATTTCATGGT GCAGTAATACCACTGTTGTTGTGTTCCTACTTTTGTGGTAAATGTGGGTGTTTAAAAAATTGTTTCTCCAACTCCTT TGAGGGTGTTCTGTGTAGAGGTTTCTTATTGGTGGCACATTITCTATCTCTTTGGAAACATGAGTGTATGAAGTGT GCATGTTGATTGCATTTTTGGCATGATGCATTAACATGTTITAAACTTCAGGCCCTTCTACTGCCAAGGTGAGTTC AGGCTGGGCGGCTGCACCCCTGGGAGCAGGGCAGTGCTGCACTGAGCCAGGCGGGAGCTGGAAGAAGACG CAGCACACTGGG

Luc-miR-744-C AAGATCTCCCCTGAACAACACTGGGATTTCACTGCAGAGGACTTGAAAGACCTTGGAGAAATTGGACGAGGAG CTTATGGTTCTGTCAACAAAATGGTCCACAAACCAAGTGGGCAAATAATGGCAGTTAAAGTAGGTGATGCCATGA TTATTTTTGGTACTTTAATCCATTAGGTGAAATTTCATGGTGCAGTAATACCACTGTTGTTGTGTTCCTACTTTTGTG GTAAATGTGGGTGTTTAAAAAATTGTTTCTCCAACTCCTTTGAGGGTGTTCTGTGTAGAGGTTTCTTATTGGTGGC ACATTTTCTATCTCTTTGGAAACATGAGTGTATGAAGTGTGCATGTTGATTGCATTTTTGGCATGATGCATTAACATG TTTTAAACTTCAGGCCCTTCTACTGCCAAGGTGAGTTCAGGCTGGGCGGCTGCACCCCTGGGAGCAGGGCAG TGCTGCACTGAGCCAGGCGGGAGCTGGAAGAAGACGCAGCACACTGGG

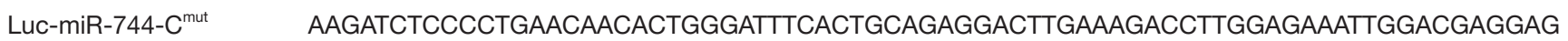
CTTATGGTTCTGTCAACAAAATGGTCCACAAACCAAGTGGGCAAATAATGGCAGTTAAAGTAGGTGATGCCATGA TTAAAAAACCTACTTTAATCCATTAGGTGAAATTTCATGGTGCAGTAATACCACTGTTGTTGTGTTCCTACTTTTGTG GTAAATGTGGGTGTTTAAAAAATTGTTTCTCCAACTCCTTTGAGGGTGTTCTGTGTAGAGGTTTCTTATTGGTGGC ACATTTTCTATCTCTTTGGAAACATGAGTGTATGAAGTGTGCATGTTGATTGCATTTTTGGCATGATGCATTAACATG TTITAAACTTCAGGCCCTTCTACTGCCAAGGTGAGTTCAGGCTGGGCGGCTGCACCCCTGGGAGCAGGGCAG TGCTGCACTGAGCCAGGCGGGAGCTGGAAGAAGACGCAGCACACTGGG

(c) Annals of Translational Medicine. All rights reserved. 